\title{
Birth prevalence of disorders detectable through newborn screening by race/ethnicity
}

\author{
Lisa Feuchtbaum, DrPH, MPH${ }^{1}$, Jennifer Carter, $\mathrm{MPH}^{2}$, Sunaina Dowray, $\mathrm{MPH}^{2}$, \\ Robert J. Currier, $\mathrm{PhD}^{1}$ and Fred Lorey, $\mathrm{PhD}^{1}$
}

Purpose: The purpose of this study was to describe the birth prevalence of genetic disorders among different racial/ethnic groups through population-based newborn screening data.

Methods: Between 7 July 2005 and 6 July 2010 newborns in California were screened for selected metabolic, endocrine, hemoglobin, and cystic fibrosis disorders using a blood sample collected via heel stick. The race and ethnicity of each newborn was self-reported by the mother at the time of specimen collection.

Results: Of 2,282,138 newborns screened, the overall disorder detection rate was 1 in 500 births. The disorder with the highest prevalence among all groups was primary congenital hypothyroidism ( 1 in 1,706 births). Birth prevalence for specific disorders varied widely among different racial/ethnic groups.

\section{INTRODUCTION}

State wide newborn screening (NBS) programs provide population-based screening for genetic disorders, with additional follow-up services to ensure that all newborns with positive screening test results are referred for confirmatory testing. In the past several years, there has been a successful nationwide effort for states to adopt a Recommended Uniform Screening Panel so that all newborns in the United States are screened for a similar set of core conditions. ${ }^{1}$ The core panel currently consists of 31 primary conditions including disorders detectable using tandem mass spectrometry (MS/MS), endocrine and hemoglobin disorders, cystic fibrosis (CF), and, recently added, severe combined immunodeficiency and critical congenital heart defects. Today, almost all states have adopted the core panel of NBS disorders, except for severe combined immunodeficiency and critical congenital heart defects, which are yet to be widely available. Additional disorders can also be identified as part of the differential diagnosis of the core panel, but these secondary disorders are not uniformly included by all state programs. The National Newborn Screening \& Genetics Resource Center provides up-to-date information about the conditions screened for within the United States. ${ }^{2}$

Despite the increase in the number of disorders detected through NBS programs in the United States, there is a lack of up-to-date knowledge about how often the disorders occur and who is affected by them. The California NBS program includes
Conclusion: The California newborn screening data offer a unique opportunity to explore the birth prevalence of many genetic disorders across a wide spectrum of racial/ethnicity classifications. The data demonstrate that racial/ethnic subgroups of the California newborn population have very different patterns of heritable disease expression. Determining the birth prevalence of these disorders in California is a first step to understanding the short- and long-term medical and treatment needs faced by affected communities, especially those groups that are impacted by more severe disorders.

Genet Med 2012:14(11):937-945

Key Words: birth prevalence; disorders; newborn screening; race and ethnicity

18 unique racial/ethnic categories on the NBS bloodspot collection card, which includes nearly all of the proposed racial/ ethnic categories described in the recently released guidelines by the US Department of Health and Human Services. ${ }^{3}$ Thus, the California NBS data offers a unique opportunity to explore the population distribution of the core and secondary disorders across racial/ethnicity classifications that closely resemble the newly promulgated categories. Determining the birth prevalence of these disorders is a first step to understanding the short- and long-term disease management needs faced by affected communities, especially those groups that are impacted by more severe disorders. These data may also be informative to public health officials in other countries who want to know which disorders impact selected populations.

Given the rarity of many of the disorders, it is difficult for individual state screening programs to develop statistically significant comparisons of disorder prevalence rates by specific racial/ ethnicity groups. Individual states may be too small or may lack significant ethnic diversity. Only limited race-specific disorder profiles have been reported elsewhere. Previous research has examined the relationship between ethnicity and a single genetic disorder, including the prevalence of a mutation or disorder within a specific ethnic group, ${ }^{4-8}$ within several ethnic groups in a region, ${ }^{9-17}$ or by geographic region only. ${ }^{18,19}$ No studies have published the disorder prevalence rates by specific racial/ethnicity groups in a large US population. California is unique in 
that it has the largest population of any single state in the United States with more than $50 \%$ of the population identified as part of a minority group. ${ }^{20}$

In July 2005, the NBS panel was expanded to include all of the primary and secondary conditions recommended by the Secretary's Advisory Committee on Heritable Disorders in Newborns and Children. ${ }^{1}$ A detailed clinical description of all of the disorders screened for can be found at the GeneReviews website. ${ }^{21}$ This article provides a comprehensive accounting of all clinical determinations for all newborns with a positive NBS test result who were referred for confirmatory testing during the study period, including those conditions with mild, questionable, or yet unknown clinical significance. Through the NBS long-term follow-up data collection process, ${ }^{22}$ we hope to learn more about the clinical significance of these and all disorders diagnosed through screening. The birth prevalence rates of these conditions (hereafter referred to as "disorders") are reported for specific racial/ethnic groups.

\section{MATERIALS AND METHODS}

The cohort described in this study includes all screened newborns who were born between 7 July 2005 and 6 July 2010. Blood specimens were collected by heel-stick between $12 \mathrm{~h}$ and 6 days after birth, with $96.6 \%$ of specimens collected between 12 and $72 \mathrm{~h}$ of age. Following collection, all specimens were sent by the collecting hospitals to a state-approved laboratory for testing. Screening for metabolic disorders was conducted by measuring acylcarnitines and amino acids by MS/MS; endocrine disorders (including congenital hypothyroidism $(\mathrm{CH})$ and congenital adrenal hypoplasia $(\mathrm{CAH})$ ) were screened by fluorescent immunoassay followed by a second-tier high-performance liquid chromatography and an tandem mass spectrometry assay for $\mathrm{CAH}$ using androstenedione, cortisol, and 17-hydroxyprogesterone; hemoglobin disorders were screened by high-performance liquid chromatography; galactosemia and biotinidase deficiency (BD) were screened for using fluorometric enzyme assays for galactose-1-phosphate uridyl transferase and biotinidase activity, respectively. CF was screened using a four-step approach (immunoreactive trypsinogen followed by a selected mutation panel, then sequencing, if the mutation panel identified two mutations, and a sweat test). ${ }^{23}$ Screening for CF and BD started on 16 June 2007 and thus, the data reported for these disorders represent a 3-year interval. Tyrosinemia type I is excluded from this report because before December 2009 the NBS panel did not include succinylacetone. Without it, screening for tyronsinemia type I has unacceptably high rates of both false positives and false negatives. Also excluded is severe combined immunodeficiency, which was started as a pilot project in the summer of 2010 .

The California NBS program screens $~ 99 \%$ of all known live births in the state. Not included are newborns born in military hospitals who are screened outside of the state program ( 5,000 births per year), births for which the parents opted out of screening ( 200 per year), and newborns who expired before screening ( $\sim 250$ per year). State law stipulates that all physicians making an initial diagnosis of a preventable heritable disorder for which screening is required shall report such a diagnosis and the information necessary for follow-up and investigation to the Public Health Department. ${ }^{24}$ Thus, this study includes all genetic disorders screened and reported in California during the 5-year time frame.

Information on race/ethnicity of the infants was reported by the mother, who was instructed to select all applicable choices from a selection of 18 distinct racial/ethnicity categories on the NBS test requisition form. To check the extent of misclassification bias of race/ethnicity, we linked NBS records with vital statistics birth certificate records for a sample year (2007) and assessed the level of agreement between the two data sources. On the basis of this analysis, we collapsed some race categories into combination groups (i.e., "Pacific Islander," "Southeast Asian") and assigned newborns to 1 of $16 \mathrm{racial} / \mathrm{ethnic}$ categories, which are defined in Table 1.

All positive test results were reported to the California Genetic Disease Screening Program (GDSP) through a secure computerbased online Screening Information System. Newborns with initial screen-positive results were referred to a state-contracted specialty follow-up center for endocrine, metabolic, hemoglobin, ${ }^{12}$ or CF disorders. Center specialists determined whether referred children had a disorder through appropriate confirmatory testing. Confirmatory algorithms for follow-up of MS/MS disorders were developed by a committee of state metabolic specialists; endocrine, hemoglobin, and CF specialists also meet regularly to review cases to promote consistency of follow-up protocols and case definitions. Final determinations of disease status were reported to the GDSP regardless of disorder severity.

Newborns who did not meet the guidelines for "typical" CF, but who were determined to have an intermediate form of the disorder called transmembrane conductance regulator-related metabolic syndrome (CRMS) were included in the list of disorders. This condition is characterized by a borderline sweat test result along with the detection of up to two cystic fibrosis transmembrane conductance regulator mutations and has been associated with a milder clinical course. ${ }^{25}$ Similarly, BD screening resulted in the diagnosis of partial $\mathrm{BD}$, the latter considered clinically less severe. ${ }^{26}$ Other more uncommon disorder variants and nonspecific categories, such as "other" amino acid/ organic acid/fatty acid oxidative disorders, are included in the Supplementary Table S1 online.

Birth prevalence rates were calculated by dividing the number of observed cases by the number of newborns screened for each disorder. To remove bias caused by genetic similarity among siblings, only the first affected case that was born to a mother during the study period was counted, and likewise only the first child born to each mother during the study period was retained in the count of newborns screened (multiple births were excluded). The observed $\mathrm{CF}$ and $\mathrm{BD}$ prevalence rates, which have been fairly constant since the initiation of screening, were multiplied by the number of newborns screened during the entire 5-year period to estimate the number of cases that would have been observed if the state had started screening for these disorders in July 2005. 
Table 1 Overall NBS disorder birth prevalence and count of screened newborns by race/ethnicity

\begin{tabular}{|c|c|c|c|}
\hline Race/ethnicity & Definition $^{a}$ & Prevalence & Screened \\
\hline Overall & All racial/ethnic groups combined & 1 in 500 & $2,282,138$ \\
\hline Southeast Asian & $\begin{array}{l}\text { Any combination of Southeast Asian categories (Cambodian, Laotian, Vietnamese, other } \\
\text { Southeast Asian) }\end{array}$ & 1 in 87 & 22,600 \\
\hline Black & Black only & 1 in 206 & 118,992 \\
\hline Multiple Asian & $\begin{array}{l}\text { Any combination of two or more Asian categories (Asian East Indian, Cambodian, Chinese, } \\
\text { Filipino, Japanese, Korean, Laotian, Middle Eastern, other Southeast Asian, Vietnamese), except } \\
\text { those already included in "Southeast Asian" }\end{array}$ & 1 in 344 & 10,669 \\
\hline Middle Eastern & Middle Eastern only & 1 in 448 & 21,973 \\
\hline Asian East Indian & Asian East-Indian only & 1 in 469 & 42,227 \\
\hline Vietnamese & Vietnamese only & 1 in 515 & 28,313 \\
\hline White & White only & 1 in 524 & 561,910 \\
\hline Hispanic & Hispanic only or Hispanic and white & 1 in 610 & $1,183,044$ \\
\hline $\begin{array}{l}\text { Hispanic- } \\
\text { multiple }\end{array}$ & $\begin{array}{l}\text { Hispanic and any other category, except those already included in "Hispanic," "Filipino," or } \\
\text { "Native American" groups }\end{array}$ & 1 in 803 & 33,737 \\
\hline Japanese & Japanese only & 1 in 961 & 5,768 \\
\hline Pacific Islander & Any combination of Pacific Islander categories (Hawaiian, Guamanian, Samoan) & 1 in 1,094 & 5,470 \\
\hline Korean & Korean only & 1 in 1,426 & 18,534 \\
\hline
\end{tabular}

aDefinitions for all racial/ethnicity categories as indicated by mother of newborn.

Using exact methods based on a binomial distribution, we report the overall disorder-specific birth prevalence rate across the entire population as well as the corresponding $99 \%$ confidence interval (CI). To determine which disorders were significantly more likely to occur within a specific racial/ethnic group, we considered only rates that fall above the upper limit of the overall 99\% CI for the disorder to be significantly higher. Racespecific rates that fall below the lower limit of the overall $99 \% \mathrm{CI}$ were not reported and are indicated with a "less than" $(<)$ symbol in Tables 2 and 3. We do report the race/ethnicity-specific prevalence rates that fell within the $99 \% \mathrm{CI}$ range. Analysis was done using SAS software, Version 9.1 of the SAS System for Windows, (SAS Institute, Cary, NC).

\section{RESULTS}

A total of 2,705,729 newborns were screened for metabolic, endocrine, and hemoglobin disorders; $1,584,475$ of these newborns were screened for $\mathrm{CF}$ and $\mathrm{BD}$. A total of 4,126 cases were identified, including 4,092 cases who were identified as a result of an initial positive screening test result. A total of 28 cases $(0.67 \%)$ were missed based on the initial screen (false negatives) but subsequently reported to the GDSP. These included $10 \mathrm{CAH}$ cases ( 5 salt wasting, 4 simple virilizing, and $1 \mathrm{CAH}$-other), $7 \mathrm{CF}$ cases, 6 primary $\mathrm{CH}$ cases, $1 \mathrm{CH}$-variant case, 2 maple syrup urine disease (MSUD) cases, 1 argininosuccinic aciduria case, and $1 \mathrm{CF}$ case also determined to have $\mathrm{CH}$. Another group of 6 cases was reported to the GDSP, but was not counted in the missed cases group: 1 MSUD and 2 CF cases who were prenatally diagnosed due to a family history; one $\mathrm{CH}$ case with trisomy 21 and other complications; and 2 methylmalonic acidemia cases ( 1 mut 0 and $1 \mathrm{cbl} \mathrm{C})$ that had borderline test results and were considered "out of range" on the basis of our screening cutoffs. An additional 49 cases were reported to the GDSP but not screened by the NBS program and are not included in this analysis: 22 cases were born outside California, and 27 cases were not screened through NBS (most were born in military hospitals). After removal of siblings and estimation of the number of CF and BD cases that would have occurred over a full 5 years of screening, the adjusted totals used for birth prevalence calculations were 4,560 cases and 2,282,138 births. Table 1 shows the overall birth prevalence for all disorders combined was 200 cases/100,000 births, or 1 in every 500 births. The table also displays the number of screened newborns within each racial/ethnic group and the overall disorder birth prevalence for each group.

Figure 1 presents the cumulative birth prevalence rates by disorder type (all metabolic, all endocrine, all $\mathrm{CF}$, and all hemoglobin combined) for each of the 16 racial/ethnic groups. Southeast Asians have the overall highest disorder rate due to the high birth prevalence of hemoglobinopathies in that group. Asian East Indians have the highest birth prevalence rate for endocrine disorders, Native Americans were disproportionately affected by CF disorders, and the birth prevalence for metabolic disorders was highest for the Middle Eastern group. 
Table 2 Birth prevalence of endocrine, cystic fibrosis, and hemoglobin disorders by racial/ethnic group per 100,000 births

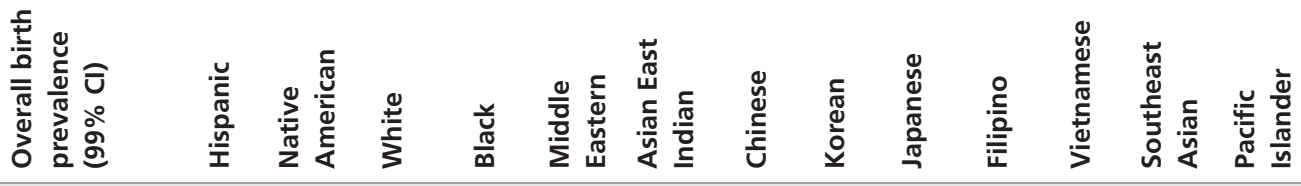

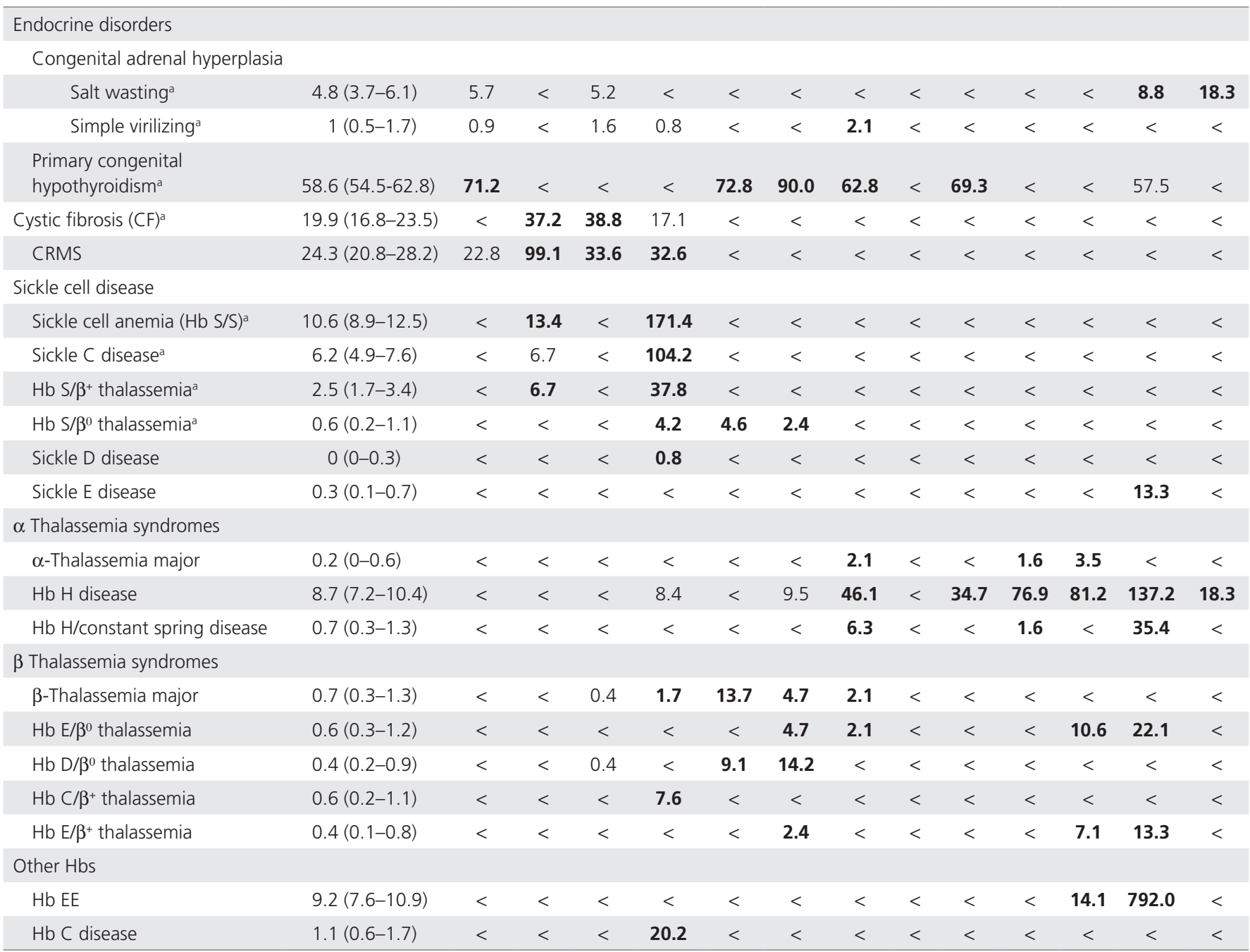

Group-specific birth prevalence rates higher than overall $99 \%$ confidence interval (CI) shown in bold; if rate falls within the $99 \% \mathrm{Cl}$, indicated in nonbold type; if less than overall $\mathrm{Cl}$, indicated as "<". CRMS, conductance regulator-related metabolic syndrome, $\mathrm{Hb}$, hemoglobin.

aRecommended Uniform Screening Panel of the Secretary's Advisory Committee on Heritable Disorders in Newborns \& Children.

Disorder-specific birth prevalence by race/ethnicity is hereafter described by specific disorder type.

Table 2 presents the overall birth prevalence rates for 21 disorders including 3 endocrine disorders, 2 CF disorders, and 16 hemoglobinopathies. Table 3 presents the overall birth prevalence rates for 42 disorders detectable by MS/MS including 12 amnio acid disorders, 16 organic acid disorders, 10 fatty acid oxidation disorders, and 4 other metabolic disorders. In both Tables 2 and 3, specific birth prevalence rates are presented for $13 \mathrm{racial} / \mathrm{ethnic}$ groups and these tables will be discussed in the following section. For a more complete list of disorders identified $(n=82)$ refer to Supplementary Table S1 online, which provides the overall birth prevalence rates, as well as the specific rates for each of the 16 racial/ethnicity groups.

\section{Endocrine disorders}

Primary $\mathrm{CH}$ was the most common of all screened disorders, with birth prevalence rates highest for six racial/ethnic groups (in order from highest to lowest rates): Asian East Indian, Middle Eastern, Hispanic, Japanese, Chinese, and Southeast Asian (Table 2). For CAH, rates that exceeded the $99 \%$ CI varied by subtype: Pacific Islanders and Southeast Asians had a higher rate for the salt-wasting type and the Chinese group had a higher rate for the simple virilizing type.

\section{CF disorders}

The overall birth prevalence for confirmed CF was 19.9 per 100,000 births, but the rate for CRMS was even higher at 24.3 per 100,000 births (Table 2). The rates among whites and Native 
Table 3 Birth prevalence of disorders detected by tandem mass spectrometry by racial/ethnic group (per 100,000 births)

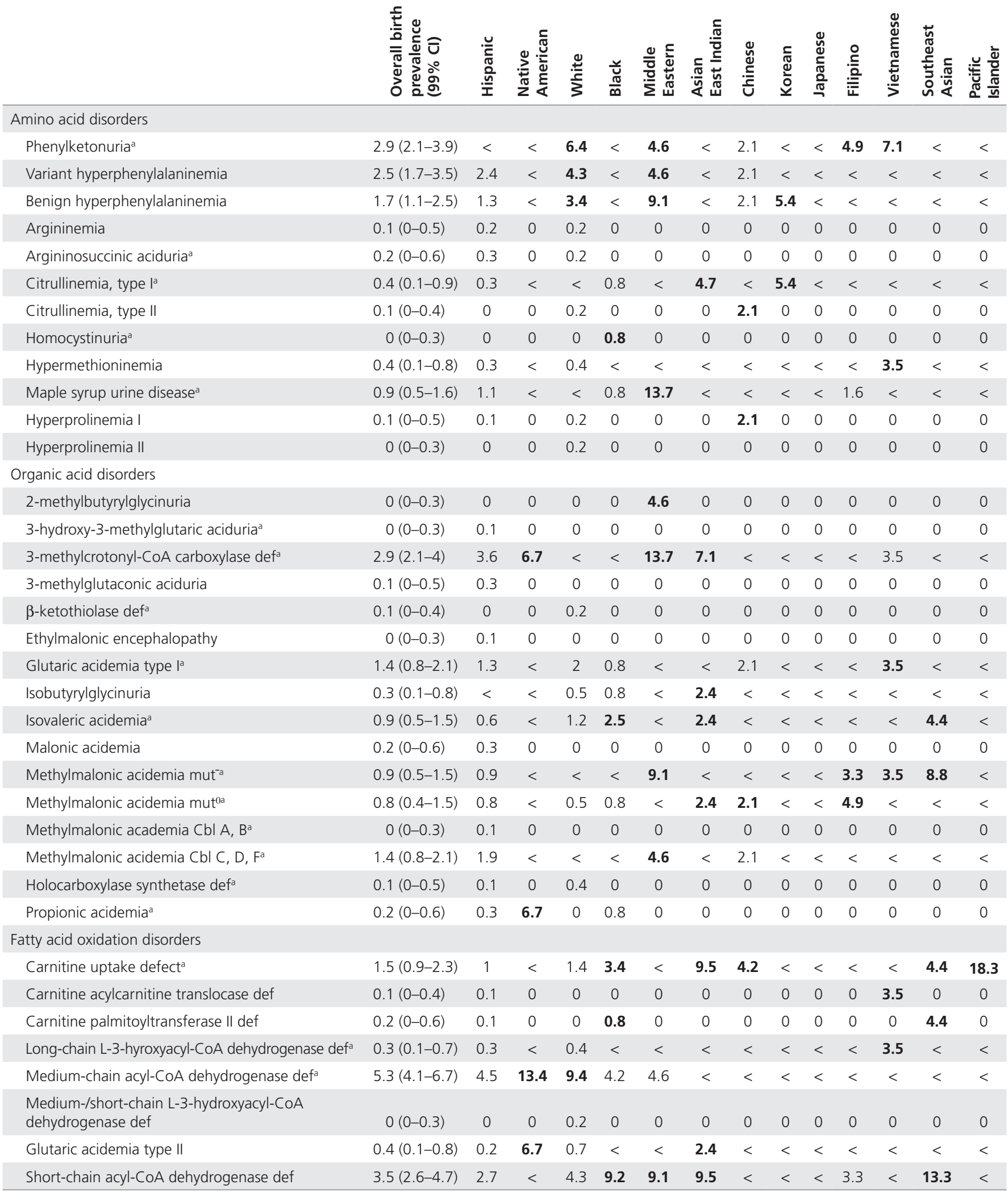

Group-specific birth prevalence rates higher than overall $99 \%$ confidence interval (CI) shown in bold; if rate falls within the $99 \% \mathrm{Cl}$, indicated in nonbold type; if less than overall $\mathrm{Cl}$, indicated as " $<$ ". def, deficiency; mut ${ }^{0}$ and mut represent complete and partial deficiency of the enzyme methylmalonyl CoA mutase.

aRecommended Uniform Screening Panel of the Secretary's Advisory Committee on Heritable Disorders in Newborns \& Children. 
Table 3 Continued

\begin{tabular}{|c|c|c|c|c|c|c|c|c|c|c|c|c|c|c|}
\hline & 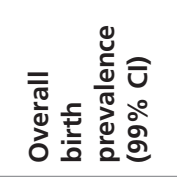 & $\begin{array}{l}. \frac{u}{2} \\
\frac{0}{\pi} \\
\frac{0}{n} \\
\frac{\underline{n}}{x}\end{array}$ & 竞 & $\frac{\stackrel{ \pm}{\frac{t}{2}}}{\frac{5}{3}}$ & $\begin{array}{l}\frac{\text { ․ }}{\infty} \\
\frac{\pi}{\infty}\end{array}$ & 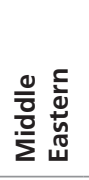 & 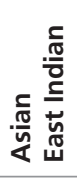 & ֻัญ & 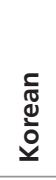 & $\begin{array}{l}\searrow \\
\dddot{y} \\
\frac{0}{\pi} \\
\stackrel{0}{0} \\
\frac{0}{2}\end{array}$ & $\frac{\circ}{\frac{2}{2}}$ & 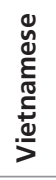 & 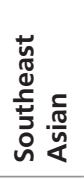 & 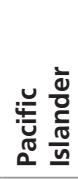 \\
\hline Trifunctional protein defa & $0(0-0.3)$ & 0 & 0 & 0.2 & 0 & 0 & 0 & 0 & 0 & 0 & 0 & 0 & 0 & 0 \\
\hline Very long-chain acyl-CoA dehydrogenase defa & $1.8(1.1-2.6)$ & $<$ & 6.7 & 2.3 & 2.5 & 4.6 & 4.7 & 2.1 & 5.4 & $<$ & 1.6 & $<$ & $<$ & $<$ \\
\hline \multicolumn{15}{|l|}{ Other metabolic disorders } \\
\hline Duarte galactosemia & $5.6(4.4-7)$ & 5.6 & $<$ & 8.9 & $<$ & 4.6 & 4.7 & $<$ & $<$ & $<$ & $<$ & $<$ & 4.4 & $<$ \\
\hline Profound biotinidase defa & $1.7(0.9-3)$ & 2.2 & $<$ & 1.7 & 1.6 & 8.4 & $<$ & $<$ & $<$ & $<$ & $<$ & $<$ & $<$ & $<$ \\
\hline Partial biotinidase defa & $2.2(1.3-3.6)$ & $<$ & $<$ & 4.9 & $<$ & 8.4 & $<$ & $<$ & $<$ & $<$ & 3 & $<$ & $<$ & $<$ \\
\hline
\end{tabular}

Group-specific birth prevalence rates higher than overall $99 \%$ confidence interval (CI) shown in bold; if rate falls within the $99 \%$ Cl, indicated in nonbold type; if less than overall $\mathrm{Cl}$, indicated as " $<$ ". def, deficiency; mut ${ }^{0}$ and mut" represent complete and partial deficiency of the enzyme methylmalonyl CoA mutase.

aRecommended Uniform Screening Panel of the Secretary's Advisory Committee on Heritable Disorders in Newborns \& Children.

Americans exceeded the overall CI for CF; these groups as well as blacks had high rates of CRMS.

\section{Hemoglobin disorders}

The highest overall birth prevalence rate of hemoglobinopathies was for sickle cell anemia, hemoglobin EE, and hemoglobin $\mathrm{H}$ disease (Table 2). Blacks had rates that exceeded the overall $\mathrm{CI}$ for 9 different hemoglobinopathies; the highest rate was for sickle cell anemia, sickle $C$ disease, and hemoglobin $(\mathrm{Hb}) \mathrm{S} / \beta+$ thalassemia. The birth prevalence for hemoglobin EE was highest among Southeast Asians (although generally considered clinically benign), and hemoglobin $\mathrm{H}$ disease was highly prevalent among Southeast Asians, Vietnamese, Filipino, Chinese, Japanese, and Pacific Islanders. Many of the Asian groups had higher rates of multiple types of thalassemia syndromes, and the Middle Eastern group had the highest rate of $\beta$ thalassemia.

\section{Metabolic disorders}

The highest overall birth prevalence for the metabolic disorders was for Duarte galactosemia, which was highest among whites (Table 3). Medium-chain acyl-CoA dehydrogenase deficiency and short-chain acyl-CoA dehydrogenase deficiency were the disorders with the next overall highest rates. The highest rates of medium-chain acyl-CoA dehydrogenase deficiency were found among Native Americans and whites, and for short-chain acylCoA dehydrogenase deficiency, the highest rates were observed among Southeast Asians, followed by the Asian East Indian, Black, and Middle Eastern groups. Other notably high birth prevalence rates are MSUD and 3-methylcrotonyl-CoA carboxylase deficiency among the Middle Eastern group and carnitine uptake defect/carnitine transport defect among Pacific Islanders.

\section{DISCUSSION}

The California NBS data offer a unique opportunity to explore the birth prevalence of many genetic disorders across a wide spectrum of race/ethnicity classifications. The data demonstrate that subsets of the California newborn population have very different patterns of heritable disease expression. Birth prevalence estimates may fluctuate over time due to diagnosis changes that will occur (based on program experience). Some children who are initially diagnosed as having a mild condition or who are minimally affected may be eventually reclassified as unaffected, and in some cases delayed confirmatory testing can lead to reclassification as another disorder. As we collect additional years of data, these birth prevalence estimates will become more robust. Although California providers are legally required to report diagnosed cases that are screened for in the NBS program, we have no way to know about cases that have not been reported and these estimates should therefore be considered conservative.

Regarding the $0.67 \%$ missed-case rate, we excluded six known cases including three prenatally diagnosed cases, and two methylmalonic acidomia (MMA) cases-one MMA (cobalamin C) and one MMA (mut-) that had borderline, or "out of range" test results. However, we included two MSUD cases, both of which were noted to have a late-onset variant, confirming that NBS may fail to identify variant forms of MSUD..$^{27}$ Total parenteral nutrition can be a major confounder when trying to interpret the results of MS/MS screen-positive cases, but the two MSUD missed cases and the one argininosuccinic aciduria case were all in the regular nursery and not on total parenteral nutrition. More recent program data has suggested that $\mathrm{CH}$ can be missed in premature births, but in this series only two of seven $\mathrm{CH}$ cases were in the neonatal intensive care unit, only one of which was considered low birth weight (1,690 g). Lastly, 10 CAH cases were missed and this suggests the need for further evaluation of the screening algorithm for those disorders. If we include the six additional cases that we excluded, the missed case rate would still be well under $1 \%$.

These birth prevalence estimates from California's NBS program are frequently higher than previously reported estimates from other states or countries. California's rate of $\mathrm{CH}$ among Asian East Indians is more than twice that reported in India ${ }^{28}$ and four times as high as rates among Hispanics in Texas. ${ }^{13}$ 


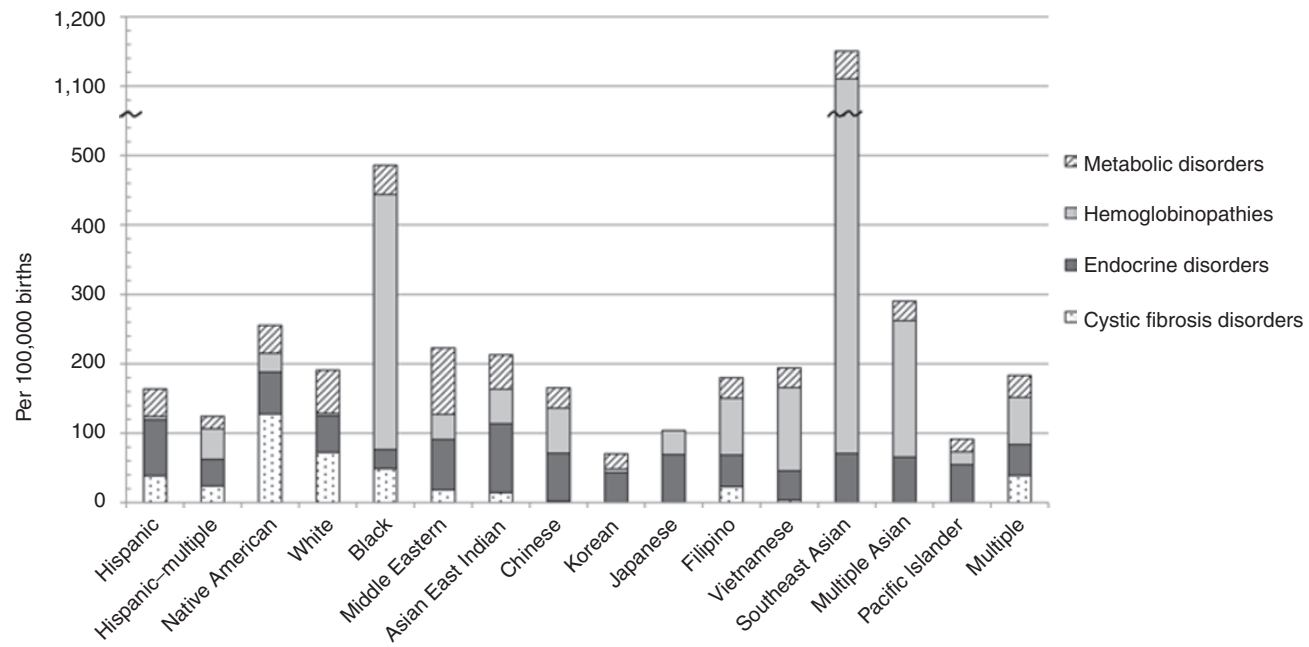

Figure 1 Cumulative California birth prevalence of all metabolic, hemoglobin, endocrine, and cystic fibrosis disorders diagnosed by race/ ethnicity $(n=2,282,138)$. Results reflect all clinical determinations reported by California state-contracted follow-up centers for women referred for confirmatory testing following a positive newborn screening test result.

For $\mathrm{CH}$ prevalence among Japanese newborns, the California estimate is slightly higher than an estimate from Japan. ${ }^{29}$ For $\mathrm{CAH}$, high rates have been reported in the Filipino population $(1: 7,000),{ }^{4}$ yet no cases were reported in our series. CF rates in California are consistent with previous reports ${ }^{18}$ in that the highest rates are in whites, however no comparison rate for the Native American group could be found. Among metabolic disorders, the rate for carnitine uptake defect among Chinese in California is higher than previously reported in a comparable population in Taiwan ${ }^{30,31}$ and likewise for methylmalonic acidomia. ${ }^{31}$ However, the prevalence of short-chain acyl-CoA dehydrogenase deficiency among whites in California is much lower than that estimated in the Netherlands. ${ }^{32}$ For hemoglobinopathies, the high rates among blacks and Asians have been previously recognized. ${ }^{33,34}$ The contrasts between prevalence estimates in California and those elsewhere reflect the complexities of defining cases as well as racial and ethnic groups.

The estimates of the birth prevalence rates for the specific racial/ethnic groups are limited by how well the data capture the true racial/ethnic identity of the child. Although NBS program protocol advises the nurse/clerk collecting the NBS specimen to use the mother's self-report of her child's race/ethnicity rather than the collectors' own observations, we cannot monitor actual collection practices. In addition, we do not know whether the mother describes her child's race/ethnicity based on her own race/ethnicity, or the father's, or a combination of both. The fact that mixed marriages are becoming more prevalent complicates this further.

One way of assessing the degree of racial/ethnicity misclassification was to compare which of the $16 \mathrm{racial} /$ ethnicity categories each newborn would have been assigned to if we had used the race/ethnicity from the birth certificate instead of NBS data. We linked all births for the year 2007 to the vital statistics birth data, with the exception of the Middle Eastern category, which is not included on the birth certificate form. Of these newborns,
$81 \%$ were assigned to the same racial/ethnic group according to both data sources. Agreement was highest (91\%) for newborns that had only a single race indicated ( $92 \%$ of NBS forms and $87 \%$ of birth certificate records). A previous study using a 1994/1995 cohort of California births also found lower concordance in mother's self-report of her race/ethnicity compared with what was recorded on the child's birth record when more than one race/ethnicity was identified..$^{35}$

The combination of race and ethnicity into a single question on the NBS data collection form leaves open the possibility that mothers may not indicate both a race and an ethnicity for their child. For example, $81.7 \%$ of newborns classified as Hispanic had no race category indicated on the NBS form. Whereas many surveys ask individuals their race in one question and whether they are of Hispanic origin in a separate question, the California NBS program does not as a result of input from Hispanic citizens.

The approach that we used to determine which racial/ethnic groups had higher birth prevalence rates than the $99 \%$ CI of the population-wide rate has limitations, especially for the Hispanic population. The overall CI is strongly influenced by the large Hispanic population, which comprises over $50 \%$ of the entire population. This leads to a diluted ability to detect higher rates of disorders among the Hispanic population because they are less likely to fall outside the $99 \%$ CI.

Analyzing disorders by racial/ethnicity classifications may cause poor health to be falsely attributed to biological or genetic factors rather than social, political, or cultural factors, as has been previously argued. ${ }^{36}$ Burchard ${ }^{37}$ criticizes the use of racial and ethnic categories as surrogates for genetic or biological markers because "an excessive focus on racial or ethnic differences runs the risk of undervaluing the great diversity that exists among persons within groups." Although these criticisms may be apt for studies examining multifactorial chronic diseases where personal behavior and environmental factors play 
a larger role, race and ethnicity data may be quite appropriate when applied to determining high-risk individuals and underlying genetic causes of disease. ${ }^{38}$

Although the birth prevalence of these disorders may be determined primarily by genetics, the outcomes and quality of life for affected children will depend on the severity of the disorder and the availability of appropriate ongoing care. The ability to obtain care throughout the lifespan will be influenced by broader socioeconomic determinants of health, which are often correlated to race and ethnicity. This includes level of education, place of residence, and type of health insurance. In addition, the community's perception of the "need" for specialty follow-up services, the costs associated with the delivery of these services, and the ability to access this care will also influence whether affected children will receive appropriate long-term follow-up care. For example, the relatively large number of individuals in the black community who are diagnosed with sickle cell disease continue to face ongoing challenges accessing high-quality disease management services necessary for improved quality of life..$^{39,40}$ New immigrant communities in California may face additional unique challenges in accessing long-term care as a result of language and cultural barriers.

In conclusion, these data highlight the fact that some population groups are more likely to bear the burden of different types of genetic disorders and will benefit from early diagnosis, which is the hallmark of a successful NBS program. However, the overall success of NBS should be assessed by the program's ability to provide ongoing long-term disease management services for affected children and their families in a culturally appropriate manner. The data from this report will provide important information about the medical and treatment needs faced by different segments of the population, especially those groups that are impacted by more and more severe disorders.

\section{SUPPLEMENTARY MATERIAL}

Supplementary material is linked to the online version of the paper at http://www.nature.com/gim

\section{ACKNOWLEDGMENTS}

S.D. and J.C. were supported by Centers for Disease Control and Prevention grant 1U50DD000475.

\section{DISCLOSURE}

The authors declare no conflict of interest.

\section{REFERENCES}

1. American College of Medical Genetics Newbond Screening Expert Group Newborn screening: toward a uniform screening panel and system. Genet Med 2006;8(suppl 1):1S-252S.

2. Lorey F, Cunningham G, Vichinsky EP, et al. Universal newborn screening for $\mathrm{Hb}$ H disease in California. Genet Test 2001;5:93-100.

3. U.S. Department of Health and Human Services. Implementation Guidance on Data Collection Standards for Race, Ethnicity, Sex, Primary Language, and Disability Status 2011. http://aspe.hhs.gov/datacncl/standards/ ACA/4302. Accessed 9 March 2012.

4. Cutiongco-de laPaz EM, Abaya EC, Silao LT, Capistrano-Estrada S, David-Padilla C. Mutations of the steroid 21-hydroxylase gene among
Filipino patients with congenital adrenal hyperplasia. Acta Med Philipp 2009;43:32-35.

5. Schrijver I. Mutation distribution in expanded screening for cystic fibrosis: making up the balance in a context of ethnic diversity. Clin Chem 2011;57:799-801.

6. Edelmann L, Wasserstein MP, Kornreich R, Sansaricq C, Snyderman SE, Diaz GA. Maple syrup urine disease: identification and carrier-frequency determination of a novel founder mutation in the Ashkenazi Jewish population. Am J Hum Genet 2001;69:863-868.

7. Souza AE, Cardoso GL, Takanashi SY, Guerreiro JF. Alpha-thalassemia (3.7 kb deletion) in a population from the Brazilian Amazon region: Santarém, Pará State. Genet Mol Res 2009;8:477-481.

8. Matern D, He M, Berry SA, et al. Prospective diagnosis of 2-methylbutyrylCOA dehydrogenase deficiency in the Hmong population by newborn screening using tandem mass spectrometry. Pediatrics 2003; 112(1 Pt 1):74-78.

9. Hutchesson AC, Bundey S, Preece MA, Hall SK, Green A. A comparison of disease and gene frequencies of inborn errors of metabolism among different ethnic groups in the West Midlands, UK. J Med Genet 1998;35:366-370

10. Lorey FW, Cunningham GC. Birth prevalence of primary congenital hypothyroidism by sex and ethnicity. Hum Biol 1992;64:531-538.

11. Waller DK, Anderson JL, Lorey F, Cunningham GC. Risk factors for congenital hypothyroidism: an investigation of infant's birth weight, ethnicity, and gender in California, 1990-1998. Teratology 2000;62: 36-41.

12. Michlitsch J, Azimi M, Hoppe $C$, et al. Newborn screening for hemoglobinopathies in California. Pediatr Blood Cancer 2009;52:486490.

13. Hinton $C F$, Harris $K B$, Borgfeld $L$, et al. Trends in incidence rates of congenital hypothyroidism related to select demographic factors: data from the United States, California, Massachusetts, New York, and Texas. Pediatrics 2010;125(suppl 2):S37-S47.

14. Bhardwaj U, Zhang YH, Lorey F, McCabe LL, McCabe ER. Molecular genetic confirmatory testing from newborn screening samples for the common African-American, Asian Indian, Southeast Asian, and Chinese beta-thalassemia mutations. Am J Hematol 2005;78: 249-255.

15. McCormick J, Green MW, Mehta G, Culross F, Mehta A. Demographics of the UK cystic fibrosis population: implications for neonatal screening. Eur J Hum Genet 2002;10:583-590.

16. Grant DB, Smith I. Survey of neonatal screening for primary hypothyroidism in England, Wales, and Northern Ireland 1982-4. Br Med I (Clin Res Ed) 1988;296:1355-1358

17. Hoppe CC. Newborn screening for non-sickling hemoglobinopathies. Hematology Am Soc Hematol Educ Program 2009;19-25.

18. Bobadilla JL, Macek M Jr, Fine JP, Farrell PM. Cystic fibrosis: a worldwide analysis of CFTR mutations-correlation with incidence data and application to screening. Hum Mutat 2002;19:575-606.

19. The Molecular Genetic Epidemiology of Cystic Fibrosis: Report of a Joint Meeting of WHO/ECFTN/ICF(M)A/ECFS, Genoa, Italy, 19 June 2002. World Health Organization, 2004

20. California. State and County QuickFacts. 2011 http://quickfacts.census. gov/qfd/states/06000.html. 2011. Accessed 26 August 2011.

21. Pagon RA, Bird TD, Dolan CR, Stephens K, (eds). GeneReviews ${ }^{T M}$ [Internet]. University of Washington: Seattle, WA, 1993. http://www.ncbi.nim.nih. gov/books/NBK1116/. Accessed 22 June 2011.

22. Feuchtbaum L, Dowray S, Lorey F. The context and approach for the California newborn screening short- and long-term follow-up data system: preliminary findings. Genet Med 2010;12(12 suppl): S242-S250.

23. California Department of Public Health GDB, Newborn Screening Program. Newborn Screening News. 2007: 15. http://www.cdph.ca.gov/ programs/nbs/Documents/NBS-NBSNewsSummer07.pdf. Accessed 25 May 2011.

24. Lorey F. Asian immigration and public health in California: thalassemia in newborns in California. J Pediatr Hematol Oncol 2000;22:564-566.

25. Ren $\mathrm{CL}$, Desai H, Platt M, Dixon M. Clinical outcomes in infants with cystic fibrosis transmembrane conductance regulator (CFTR) related metabolic syndrome. Pediatr Pulmonol 2011;46:1079-1084.

26. Wolf B. Biotinidase deficiency. In: Pagon R, Bird T, Dolan C (eds) GeneReviews. University of Washington: Seattle, 2000. 
27. Puckett RL, Lorey F, Rinaldo P, et al. Maple syrup urine disease: further evidence that newborn screening may fail to identify variant forms. Mol Genet Metab 2010;100:136-142.

28. Nair PS, Sobhakumar S, Kailas L. Diagnostic re-evaluation of children with congenital hypothyroidism. Indian Pediatr 2010;47:757-760.

29. Gu YH, Kato T, Harada S, Inomata H, Aoki K. Time trend and geographic distribution of treated patients with congenital hypothyroidism relative to the number of available endocrinologists in Japan. J Pediatr 2010;157:153-157.

30. Lee NC, Tang NL, Chien YH, et al. Diagnoses of newborns and mothers with carnitine uptake defects through newborn screening. Mol Genet Metab 2010;100:46-50.

31. Niu DM, Chien YH, Chiang CC, et al. Nationwide survey of extended newborn screening by tandem mass spectrometry in Taiwan. J Inherit Metab Dis 2010;33(suppl 2):S295-\$305.

32. van Maldegem BT, Kloosterman SF, Janssen WJ, et al. High prevalence of short-chain acyl-CoA dehydrogenase deficiency in the Netherlands, but no association with epilepsy of unknown origin in childhood. Neuropediatrics 2011;42:13-17.

33. Hassell KL. Population estimates of sickle cell disease in the U.S. Am J Prev Med 2010;38(4 suppl):S512-S521.
34. Colah R, Gorakshakar A, Nadkarni A. Global burden, distribution and prevention of B-thalassemias and hemoglobin E disorders. Expert Rev Hematol 2010;3:103-117.

35. Baumeister L, Marchi K, Pearl M, Williams R, Braveman P. The validity of information on "race" and "Hispanic ethnicity" in California birth certificate data. Health Serv Res 2000;35:869-883.

36. Rebbeck TR, Sankar P. Ethnicity, ancestry, and race in molecular epidemiologic research. Cancer Epidemiol Biomarkers Prev 2005;14(11 Pt 1):2467-2471.

37. Burchard EG, Ziv E, Coyle N, et al. The importance of race and ethnic background in biomedical research and clinical practice. N Engl J Med 2003;348:1170-1175.

38. Kahn J. Genes, race, and population: avoiding a collision of categories. Am J Public Health 2006;96:1965-1970.

39. Boulet SL, Yanni EA, Creary MS, Olney RS. Health status and healthcare use in a national sample of children with sickle cell disease. Am J Prev Med 2010;38(4 suppl):S528-S535.

40. Prabhakar H, Haywood C Jr, Molokie R. Sickle cell disease in the United States: looking back and forward at 100 years of progress in management and survival. Am J Hematol 2010;85: 346-353. 